\title{
Accesibilidad y utilización de servicios de salud en hombres con discapacidad motora
}

\author{
Accessibility and use of health care services for men with motor disability \\ Acessibilidade e utilização de serviços de saúde em homens com incapacidade motora
}

\author{
Kyara Herrera-Pamplona* \\ Irma Yolanda Castillo-Ávila**
}

\begin{abstract}
Autor de correspondencia
* Enfermera. Universidad de Cartagena. Facultad de Enfermería. Correo: kherrerap1@unicartagena.edu.co. Orcid: (iD https:// orcid.org/0000-0002-6969-1212. Cartagena de Indias, Colombia

* Enfermera, Magíster en Salud Pública. Docente Universidad de Cartagena, Facultad de Enfermería. Correo: icastilloa@unicartagena.edu.co. Orcid: (D) https://orcid. org/0000-0002-4423-0874. Cartagena de Indias, Colombia
\end{abstract}

\section{Resumen}

Objetivo: Determinar los factores asociados a la accesibilidad y utilización de servicios de salud preventivos en hombres con discapacidad motora de una localidad de la ciudad de Cartagena. Materiales y Métodos: Estudio analítico de corte transversal, población de referencia de 1.661 adultos con discapacidad motora. La muestra fue de 204 hombres con discapacidad motora. Muestreo aleatorio estratificado. Para la recolección de la información se aplicó un instrumento que incluyó una ficha sociodemográfica, ficha de uso, diseñada por los autores, cuestionario APGAR familiar (fiabilidad de 0.79), cuestionario de apoyo social Duck 11 (alfa de Cronbach de 0,90) además la escala para medir la satisfacción (fiabilidad de 0.82). La información obtenida se procesó en Microsoft Excel y fue analizada en el programa estadístico SPSSS versión 20. Resultados: Edad promedio 45 años ( $\mathrm{DE}=12,9$ años), el 72,5\% (148) proceden del área urbana, 44,5\% (90) tiene pareja, 59\%(122) ha tenido una consulta en los últimos 6 meses y solo 6,5\%(8) asiste por estar inscrito a programas de promoción y prevención. El 52,5\% (107) considera que el centro de salud queda lejos de su vivienda, $53 \%$ (108) espera para ser atendido desde de 30 a 60 minutos, 55,4\% (113) espera más de 7 días para asignación de citas médicas. Conclusiones: El uso de servicios de salud preventivos está mediado por determinantes personales como tener una pareja y estudios superiores a la secundaria; así mismo, el apoyo social, la satisfacción con el acceso y la infraestructura de los centros de salud facilitan la utilización de estos servicios.

Palabras clave: Personas con discapacidad, accesibilidad a los servicios de salud, servicios de salud, salud pública.

\section{Abstract}

Objective: To determine the factors associated with the accessibility and use of preventive health services in men with motor disabilities in a town in the city of Cartagena. Materials and methods: Analytical cross-sectional study, reference population of 1,661 adults with motor disability. The sample was 204 men with motor disability. Stratified random sampling. For the collection of information, an instrument was applied
Este es un artículo bajo la licencia CC BY

(https://creativecommons.org/ licenses/by/4.0/) @ (1)

Doi: https://doi.org/10.22463/17949831.1543

Recibido: 13 de Septiembre 2018. Aprobado: 12 de Diciembre 2018

Para citar este artículo/ To reference this article/ Para citar este artigo/

Herrera Pamplona K, Castillo Ávila IY. Accesibilidad y utilización de servicios de salud en hombres con discapacidad motora. Rev. cienc. cuidad. 2019; 16(1):32-46 
that included a sociodemographic record, use tab, designed by the authors, Family APGAR Questionnaire (reliability of 0.79 ), social support questionnaire Duck 11 (Cronbach's alpha of 0.90) plus the scale to measure satisfaction (reliability of 0.82 ). The information obtained was processed in Microsoft Excel and analyzed in the statistical program SPSSS version 20. Results: Average age 45 years (SD $=12.9$ years), $72.5 \%$ (148) come from the urban area, $44.5 \%$ (90) has a partner, $59 \%(122)$ has had a consultation in the last 6 months and only $6.5 \%(8)$ attends to be enrolled in promotion and prevention programs. $52.5 \%$ (107) consider that the health center is far from their home, $53 \%(108)$ expected to be attended from 30 to 60 minutes, 55.4\% (113) expected more than 7 days for assignment of medical appointments. Conclusions: The use of preventive health services is mediated by personal determinants such as having a partner and higher education than the secondary one; Likewise, social support, satisfaction with access and the infrastructure of health centers facilitate the use of these services.

Key words: Disabled Persons, Health Services Accessibility, health services, Public health (Source: $\mathrm{MeSH})$.

\begin{abstract}
Resumo
Objetivo: Determinar os fatores relacionados com a intenção de desertar em estudantes de enfermagem de uma universidade pública na Colômbia. Materiais e Métodos: Estudo de corte transversal analítico. A amostra correspondeu a 162 estudantes dos diferentes níveis acadêmicos. Para identificar os fatores de risco se criou um questionário com 54 itens que se someteu a validade facial, e incluiu uma pergunta para estabelecer a intenção de desertar do curso no último mês. Realizouse uma análise descritiva e se construiu um modelo de regressão logística que identificou os fatores relacionados com a presença de intenção de desertar do curso de enfermagem. A análise estadística se realizou no programa Stata v12 software. Resultados: A média de idade dos participantes foi de 21 anos, o 85\% pertenceram ao género feminino; o 20\% $(n=32)$ declarou haver considerado desertar do programa de enfermagem no último mês, destes 78\% (25/32) cursavam sexto ou níveis inferiores. Os fatores de risco para a intenção de desertar foram: sintomas depressivos de ansiedade e depressão; falta de interesse nas disciplinas do programa relacionado com ausência de identidade vocacional; relacionamento regular com os professores e pertencer a baixo estrato socioeconómico. Conclusões: $\mathrm{O}$ interesse pelas disciplinas e o relacionamento com os professores estão relacionados com a intenção de desertar, assim como, o componente de saúde mental e o estrato socioeconômico. Requerem-se estudos longitudinais para corroborar estas descobertas.
\end{abstract}

Palavras-chave: Educação superior, deserção, fatores de risco.

\section{Introducción}

Más de mil millones de personas viven en todo el mundo con alguna forma de discapacidad, de ellas, aproximadamente el 20\% manifiestan alteraciones que afectan de manera considerable diferentes aspectos de su vida. La discapacidad es un problema de salud pública debido al incremento que ha tenido su prevalencia en los últimos años, se prevé que en un futuro sea un motivo de mayor preocupación, teniendo en cuenta que la población está envejeciendo y el riesgo de discapacidad es alto entre los adultos mayores (1-3).

Existen diferentes tipos de discapacidad, dentro de los cuales está la discapacidad motora, definida como la dificultad que presenta una persona en el manejo y control de su cuerpo, y que por sus características necesita y requiere de diversos elementos para des- envolverse. Se considera una persona con discapacidad motora cuando por causas cerebrales, nerviosas, musculares y/o físicas se ve afectada su habilidad en el control y manejo del equilibrio, postura, coordinación y movimiento de las diferentes partes del cuerpo $(4,5)$.

En Colombia, de acuerdo al Departamento Administrativo Nacional de Estadística (DANE) la población total de personas con discapacidad se encuentra alrededor de 2.624 .898 personas lo que equivale al $6,3 \%$ del total de la población. Las alteraciones de movimiento del cuerpo, manos, brazos, piernas se identifican como las afecciones fisiológicas más frecuentes; con un $22 \%$ en 2005 y un $30.8 \%$ en 2011. Según el Registro para la localización y caracterización de las personas con discapacidad (RLCPD) del país en el año 2013 se identificaron 981.181 personas con discapacidad, cifra que a la fecha correspondía al 37,4\%. En el distrito de Cartagena 
ISSN-PRINT

1794-9831

E-ISSN 2322-7028

Vol. 16 No. 1

Ene - Abr 2019

Cúcuta, Colombia el RLCPD, se identificaron 5.210 personas con discapacidad motora, lo que corresponde a un $46,8 \%$ de la población total con discapacidad de la ciudad $(3,6,7)$. Así mismo, en la ciudad se observa que solo el $72,7 \%$ de las personas con discapacidad están afiliadas al Sistema de Salud, es decir, que una de cada tres personas, se encuentra excluida de los servicios de salud y por tanto, este grupo presenta una mayor vulnerabilidad respecto al total de la población $(7,8)$. El $96,6 \%$ de la población con discapacidad de la Ciudad, según datos del SISBEN se encuentra en los niveles 1 y 2 ; un elevado porcentaje $(65,47 \%)$ se ubica en el nivel 1 , lo que además muestra la crítica situación socioeconómica de este grupo poblacional (9).

Las personas con discapacidad tienen una mayor demanda en asistencia sanitaria y mayores necesidades en salud respecto a quienes no tienen una discapacidad. En Colombia la situación de las personas con discapacidad, muestra que a pesar de la extensa normatividad y las actividades desarrolladas para promover la inclusión de estas, aún existen limitaciones en el acceso a actividades de promoción de la salud y prevención de la enfermedad, donde sólo en raras ocasiones se tiene como destinatario a este grupo poblacional. Por ejemplo en programas de educación sexual, los adolescentes y adultos con discapacidades quedan excluidos más a menudo $(10,11)$.

La Constitución Política de 1991, la Ley 100 de 1993 y la Ley 60 del mismo año contribuyeron en la reforma del Sistema general de Seguridad social en Salud de Colombia, principalmente en lo relacionado con brindar un servicio de salud más equitativo y solidario para todos los ciudadanos. Es por eso, que quienes tienen mayores ingresos, subsidian y/o auxilian a los ciudadanos con menos ingresos. Sin embargo, existen falencias en la vinculación total de las personas con discapacidad al Sistema de Salud en Colombia. De ahí, que corresponda al estado asegurar la prestación de atención médica eficaz para las personas con discapacidad, aunque no se tiene una cobertura en el sistema de salud para todas las personas con discapacidad $(12,13)$.

El aseguramiento adoptado por la Ley 100 de 1993, reconoce al afiliado el derecho a ser atendido mediante un paquete de servicios cuando lo requiera, gozando de la mayor equidad posible y sin ningún tipo de discriminación. La Resolución 3165 de 1996 y el Decreto 1538 de 2005 son normas mediante las cuales se busca garantizar la accesibilidad a las personas con discapacidad y se adoptan lineamientos de atención en salud para que las personas con deficiencias, discapacidades y minusvalías, sean respaldadas desde el marco legal. Sin embargo, en la práctica, la población en general enfrenta múltiples barreras de tipo geográfico, normativo, administrativo, cultural y de oferta para acceder a los servicios de salud. (14-17)

Por otra parte, las personas con discapacidad que se encuentran afiliadas al sistema de salud recurren, la gran mayoría de las veces, a una acción de tutela para acceder de forma total, incluso parcial a los servicios de salud y al cumplimiento de sus derechos en salud (18); esto debido a los altos costos en procesos de rehabilitación, intervenciones tempranas, vigilancia de condiciones crónicas y el uso de tecnología $(12,19)$.

Estudios realizados sobre este tipo de limitación física, han demostrado que las personas con alguna condición de discapacidad tienen: peores niveles de salud que la población en general, resultados académicos más bajos, menor participación en la vida familiar, social y comunitaria, y menos ingresos económicos; además pueden experimentar mayor vulnerabilidad a enfermedades prevenibles, trastornos relacionados con la edad, y comorbilidades secundarias a su condición de discapacidad (1).

Existen servicios de salud preventivos disponibles para toda la población y estos deben ser garantizados a todos los ciudadanos. Sin embargo, las personas con discapacidad a pesar de tener una mayor demanda de asistencia sanitaria, frente a quienes no la padecen y mayores necesidades de salud, son quienes menos utilizan estos servicios, lo cual se traduce en un mayor riesgo para el padecimiento de este tipo de enfermedades fácilmente prevenibles $(10,20)$.

El acceso a los servicios de salud ha sido abordado por muchos autores; no obstante, las investigaciones se han centrado especialmente en la población en general. Los servicios de salud poseen características que facilitan u obstaculizan la utilización y accesibilidad de los mismos. Específicamente en el tema de la discapacidad se ha encontrado que las principales barreras relacionadas con los servicios en salud son: la falta de calidad, calidez e integralidad de la atención en salud, la pobre cobertura de los servicios, la creencia de que la rehabilitación es un proceso costoso para el asegurador, la falta de inclusión de las ayudas técnicas en el Plan Obligatorio de Salud (POS), la escasez de profesionales capacitados, las barreras arquitectónicas de los centros que prestan ser- 
vicios de salud, el tiempo de espera y la distancia desde el hogar al establecimiento de salud. También influyen las actitudes negativas de la sociedad, que muchas veces resultan en una baja percepción de la satisfacción con estos servicios e inciden de manera directa en la percepción que construye el individuo después del encuentro e interacción con los mismos, y que lo llevan a estar satisfecho o no. En el caso de las personas con discapacidad motora cuando buscan asistencia sanitaria, en conjunto, estas características tienen repercusiones directas sobre el uso y accesibilidad de servicios ofertados por diferentes instituciones de salud $(2,10,21)$.

De igual forma, para garantizar el acceso y utilización de servicios de salud a las personas con discapacidad se requiere que los bienes y las oportunidades de salud, sean adecuados para toda la población sin importar sus condiciones físicas, teniendo en cuenta que la prestación de estos servicios a este grupo poblacional, además de ser un servicio social, es un derecho humano fundamental (7). En la ciudad de Cartagena los servicios de salud preventivos se ofrecen a toda la población; sin embargo, no se ha encontrado información estadística que permita diferenciar la utilización y accesibilidad de estos servicios por parte de la población masculina con discapacidad motora.

Es por eso, que esta situación suscita el interés por identificar los determinantes del acceso y utilización de servicios de salud preventivos por parte de hombres adultos con discapacidad motora, en una localidad de la ciudad de Cartagena. Ampliar el conocimiento sobre el acceso y utilización de los servicios de salud en hombres con discapacidad motora se constituirá en una base teórica para posteriores investigaciones en torno al tema.

Así mismo, el desarrollo de este estudio permitirá contar con información que ayude a generar y desarrollar propuestas de atención e investigación, encaminadas a mejorar la calidad de vida de esta población, mediante la creación de estrategias que cubran la gran demanda de necesidades en salud que poseen.

Es por eso que, para los profesionales de enfermería, este estudio se constituye en una herramienta útil e imprescindible, debido a que permitirá fortalecer el rol que cumple la enfermería en los programas de promoción de la salud y la prevención de la enfermedad de toda la población. Del mismo modo, permitirá impulsar estrategias que impulsen la utilización de estos servicios por parte de las personas con discapacidad, adaptándolos a los rasgos propios de este grupo poblacional; igualmente, permitirá promover una atención integral, disminuyendo así la morbimortalidad en esta población; pero también que se implementen acciones encaminadas a garantizar la equidad en el acceso a la atención en salud preventiva.

\section{Objetivos}

\section{Objetivo General}

Determinar los factores asociados a la accesibilidad y utilización de servicios de salud preventivos en hombres con discapacidad motora de la localidad 2 de la virgen y turística de la ciudad de Cartagena.

\section{Objetivos específicos}

- Identificar las características sociodemográficas de hombres con discapacidad motora de la localidad 2 de la virgen y turística de la ciudad de Cartagena.

- Describir la frecuencia de utilización de los servicios de salud preventivos por los adultos con discapacidad motora.

- Describir el acceso a los servicios de salud preventivos por los adultos con discapacidad motora.

- Estimar la asociación estadística entre los factores estudiados y la utilización de servicios preventivos.

\section{Materiales y Métodos}

Este es un estudio analítico con diseño de corte transversal, con una población de estudio de $1.661 \mathrm{y}$ un tamaño de muestra estimado de 204 hombres con discapacidad motora de la localidad 2 de la ciudad de Cartagena que aceptaron realizar la encuesta, con un $95 \%$ de confiabilidad y un $5 \%$ de error de estimación.

Se utilizó un muestreo aleatorio estratificado; cada estrato estuvo representado por los 28 barrios y 6 corregimientos de la localidad, los cuales se colocaron en una lista y al azar se seleccionaron 14. La muestra fue tomada de la localidad 2: de la virgen y turística ciudad de Cartagena, mediante afijación proporcional, de acuerdo al tamaño de la población en cada estrato.

Los criterios de inclusión fueron: adultos hombres con 
ISSN-PRINT

1794-9831

E-ISSN 2322-7028

Vol. 16 No. 1

Ene - Abr 2019

Cúcuta, Colombia discapacidad motora con edad igual o mayor a 18 años residentes en la localidad 2 de la virgen y turística ciudad de Cartagena. Los criterios de exclusión fueron: adultos hombres, que además de la discapacidad motora tuvieran limitaciones para la comunicación o enfermedades mentales previamente diagnosticadas.

La recolección de la información se realizó a través de una encuesta auto-administrada que permitió indagar sobre el acceso y uso de los servicios considerados como preventivos o de promoción de la salud, tales como: consulta médica anual, examen de agudeza visual y atención preventiva en salud oral, razón por la cual se incluyó un instrumento que constaba de una ficha sociodemográfica y una ficha de uso de servicios, diseñada por los autores, siguiendo los parámetros establecidos en la Encuesta Nacional de Demografía y Salud de Colombia 2010, para la caracterización de los hombres participantes.

Se utilizó el Cuestionario Apgar familiar que consta de 5 ítems tipo likert para valorar la percepción de la persona sobre la función familiar. Los puntos de corte son los siguientes: Normofuncional: 7 - 10 puntos, Disfuncional leve: $3-6$, Disfuncional grave: $0-2(22)$, tiene una fiabilidad de 0.79

También, se utilizó el cuestionario de apoyo social Duck 11 , que consta de 11 ítems y una escala de respuesta tipo likert (1-5). El rango de puntuación oscila entre 11 y 55 puntos. La puntuación obtenida es un reflejo del apoyo percibido, no del real, indicando que a menor puntuación, menor apoyo. En la validación española se optó por un punto de corte en el percentil 15, que corresponde a una puntuación $<32$. Una puntuación igual o mayor a 32 indica un apoyo normal, mientras que menor a 32 muestra un apoyo social percibido bajo (23), con un alfa de Cronbach de 0,90 (24).

Cabe señalar que la Escala para medir satisfacción de los servicios sanitarios fue desarrollada por Jesús Varela Mallou y Antonio Rial Boubeta. Es una escala breve y de fácil aplicación, consta de 11 ítems que evalúa el personal sanitario (4 ítems), personal de apoyo (2 ítems), condiciones físicas ( 3 ítems) y el acceso al servicio ( 2 ítems); tiene una fiabilidad de 0.82 (25). Todo lo anterior acompañado de un formato de consentimiento informado.

Se visitó casa por casa en cada uno de los barrios previamente seleccionados y se aplicó la encuesta en los domicilios donde había hombres que cumplían con los criterios de selección. Se les dio a conocer el objetivo del trabajo informándoles que sería anónimo y confidencial, y si manifestaban su deseo voluntario de participar, se les entregaba la encuesta junto con el consentimiento para su diligenciamiento. Una investigadora permanecía con la persona para resolver cualquier inquietud con respecto al cuestionario, dando un tiempo prudente de 15-20 minutos para la resolución del mismo.

La información obtenida se procesó en una hoja de cálculos del programa Microsoft Excel 2010, luego fue analizada estadísticamente mediante tablas de frecuencia y representaciones gráficas con porcentajes obtenidos con este mismo programa, exponiendo ordenadamente la información recogida en la muestra. Además se aplicó estadística inferencial para estimar las asociaciones entre las variables, utilizando el software SPSSS versión 20

El plan de análisis se centró en dos variables dependientes: utilización de servicios de salud preventivos y consulta médica en el último año e inscripción a programas de promoción y prevención. Las variables sociodemográficas y las relacionadas con la percepción de la satisfacción, así como las características del servicio se consideraron como variables independientes.

Posterior al análisis uni-variado, fue adelantado un análisis bi-variado y un modelo de regresión logística para identificar la relación entre las variables de estudio y definir aquellas que explican la utilización de los servicios preventivos.

Conviene precisar que la clasificación del estudio es de riesgo mínimo, según la Resolución No. 8430 de 1993 del Ministerio de Salud (26) y se tuvo en cuenta, además, la declaración de Helsinki y lo dispuesto por el Consejo de Organizaciones de las Ciencias Médicas (CIOMS) relacionado con las pautas éticas internacionales para la investigación biomédica en seres humanos (Principios bioéticos; autonomía, no mal eficiencia y beneficencia) (27). Así mismo, esta investigación fue aprobada por el comité de investigaciones de la facultad de enfermería de la Universidad de Cartagena.

\section{Resultados}

Características sociodemográficas de los participantes del estudio.

En el estudio participaron 204 hombres, con una 
edad promedio de 45 años (DE= 12,9 años), 72,5\% (148) proceden del área urbana; con relación al estado civil un 44,5\% (90) tenían pareja; el 27\% (55) cursaron el bachillerato completo, solo el 3,9\% (8) tiene estudios universitarios; $84,3 \%$ (172) pertenecen al estrato socioeconómico 1 . En cuanto a la ocupación, el 58,9\% (120) se encuentra trabajando y de estos el 46,2\% (94) lo hacen de manera independiente o en la modalidad informal, el 36,7\% (75) obtiene ingresos inferiores a un salario mínimo legal vigente (SMLV), solo el 11,3\%(23) recibe pensión y el $77,4 \%$ (158) se encuentra afiliado al régimen subsidiado. De acuerdo al tipo de familia predominó la familia nuclear en un $29,4 \%$ (60) y $58,8 \%$ (120) tiene apoyo social normal.

\section{Percepción de la satisfacción con los servicios médi- cos recibidos por adultos con discapacidad motora.}

Con respecto a la satisfacción de los servicios de salud preventivos, más del $50 \%$ del total de participantes respondieron el estar satisfechos con el tiempo que le dedica su médico, el trato recibido por el personal de salud, la disposición del personal por ayudarlo y las instalaciones del centro de salud. Sin embargo, un alto porcentaje de los participantes manifestó estar insatisfecho con las siguientes características: el 50 \% (102) con la solución que el médico/enfermera le da, $53 \%$ (108) con el interés del médico en explicarle la dolencia, 53,4\% (109) con la información sobre el tratamiento, 73\% (149) con la comodidad y amplitud de la sala de espera y el $67,2 \%$ (137) con la señalización (Ver Tabla 1).

Tabla 1. Satisfacción de los servicios de salud preventivos en adultos con discapacidad motora de la localidad 2 de la virgen y turística de la ciudad Cartagena.

\begin{tabular}{|c|c|c|}
\hline Característica & Frecuencia & Porcentaje \\
\hline \multicolumn{3}{|l|}{ Tiempo médico } \\
\hline Satisfecho & 111 & 54,4 \\
\hline Insatisfecho & 93 & 45,6 \\
\hline \multicolumn{3}{|c|}{ Solución médico/enfermera le da } \\
\hline Satisfecho & 102 & 50 \\
\hline Insatisfecho & 102 & 50 \\
\hline \multicolumn{3}{|c|}{ Interés médico en explicarle la dolencia } \\
\hline Satisfecho & 96 & 47,1 \\
\hline Insatisfecho & 108 & 52,9 \\
\hline \multicolumn{3}{|c|}{ Información sobre el tratamiento } \\
\hline Satisfecho & 95 & 46,6 \\
\hline Insatisfecho & 109 & 53,4 \\
\hline \multicolumn{3}{|c|}{ Trato recibido por el personal } \\
\hline Satisfecho & 109 & 53,4 \\
\hline Insatisfecho & 95 & 47,1 \\
\hline \multicolumn{3}{|c|}{ Disposición del personal para ayudar } \\
\hline Satisfecho & 108 & 52,9 \\
\hline Insatisfecho & 96 & 47,1 \\
\hline \multicolumn{3}{|c|}{ Comodidad y amplitud de la sala de espera } \\
\hline Satisfecho & 55 & 27 \\
\hline Insatisfecho & 149 & 73 \\
\hline \multicolumn{3}{|c|}{ Instalaciones del centro } \\
\hline Satisfecho & 143 & 70,1 \\
\hline Insatisfecho & 61 & 29,9 \\
\hline \multicolumn{3}{|l|}{ Señalización } \\
\hline Satisfecho & 67 & 32.8 \\
\hline Insatisfecho & 137 & 67,2 \\
\hline
\end{tabular}

Fuente: Resultados del estudio 
ISSN-PRINT

1794-9831

E-ISSN 2322-7028

Vol. 16 No. 1

Ene - Abr 2019

Cúcuta, Colombia
Frecuencia de utilización de servicios de salud preventivos por hombres adultos con discapacidad motora de la localidad 2 de Cartagena

En cuanto a la frecuencia con la que los pacientes asisten a una consulta médica al año, el 57,8\% (118) asistió de 1-2 veces al año, el 59\% (122) tuvo una consulta para el cuidado de su salud en los últimos 6 meses y solo un 6,5\% (8) asistieron por estar inscritos a un programa de promoción y prevención. El 77\% (157) no se realizó el examen de agudeza visual en el último año y el 82\% (167) no asistió a una cita odontológica en los últimos 6 meses. (Ver Tabla 2).

Tabla 2. Frecuencia de utilización de servicios de salud en hombres con discapacidad motora de la localidad 2 de la ciudad de Cartagena.

\begin{tabular}{|c|c|c|}
\hline Característica & Frecuencia & Porcentaje \\
\hline \multicolumn{3}{|c|}{ Frecuencia con la que asiste a una consulta médica al año } \\
\hline $1-2$ veces & 118 & 57,8 \\
\hline $3-4$ veces & 57 & 28 \\
\hline Más de 4 veces & 29 & 14,2 \\
\hline \multicolumn{3}{|c|}{ Consulta para el cuidado de su salud en los últimos 6 meses } \\
\hline $\mathrm{Si}$ & 122 & 59 \\
\hline No & 82 & 41 \\
\hline \multicolumn{3}{|l|}{ Motivo consulta médica } \\
\hline Programa P y P & 8 & 6,5 \\
\hline Urgencia & 31 & 25,5 \\
\hline Rutina & 83 & 68 \\
\hline \multicolumn{3}{|c|}{ Razón por la cual no solicitó o recibió atención médica } \\
\hline Caso leve & 68 & 139 \\
\hline Falta de tiempo & 5,3 & 11 \\
\hline Servicio queda lejos & 13 & 26 \\
\hline Transporte muy costoso & 0,5 & 1 \\
\hline Servicio muy costoso & 3 & 6 \\
\hline Servicio de mala calidad & 0,5 & 1 \\
\hline Fue pero no lo atendieron & 3,2 & 7 \\
\hline No confía en médicos & 0,5 & 1 \\
\hline Muchos trámites & 0,5 & 1 \\
\hline No Sabe Dónde Prestan El Servicio & 4 & 8 \\
\hline Otra & 1,5 & 3 \\
\hline \multicolumn{3}{|c|}{ Inscripción programas de promoción y prevención } \\
\hline $\mathrm{Si}$ & 19 & 9,3 \\
\hline No & 185 & 90,7 \\
\hline \multicolumn{3}{|c|}{ Examen de agudeza visual en los últimos 12 meses } \\
\hline $\mathrm{Si}$ & 47 & 23 \\
\hline No & 157 & 77 \\
\hline \multicolumn{3}{|c|}{ Citas odontológicas en los últimos 6 meses } \\
\hline $\mathrm{Si}$ & 37 & 18 \\
\hline No & 167 & 82 \\
\hline
\end{tabular}

Fuente: Resultados del estudio

Acceso a los servicios de salud preventivos por hombres adultos con discapacidad motora de la localidad 2 de Cartagena. Cuando se les preguntó a los participantes sobre la ubicación del centro de salud, el $52,5 \%$ (107) respondió que este se encuentra lejos de su vivienda, el 84,7\% (162) utilizó transporte pú- blico como medio de desplazamiento. El 53\% (108) espera para ser atendido desde que llega a la institución de salud de 30 a 60 minutos y más; en cuanto a la asignación de citas médicas, el 55,4\% (113) manifiestó que es superior a 7 días. (Ver Tabla 3) 
Tabla 3. Acceso a los servicios de salud Preventivos por hombres adultos con discapacidad motora de la localidad 2 de Cartagena

\begin{tabular}{lcc}
\hline Característica & Frecuencia & Porcentaje \\
\hline Tiempo de espera & & \\
0 -30 minutos & 96 & 47,1 \\
$30-60$ minutos & 74 & 36,3 \\
Más de 60 minutos & 34 & 16,7 \\
\hline Ubicación centro de salud & 28 & 13,7 \\
Muy lejos & 107 & 52,5 \\
Lejos & 69 & 33,8 \\
Cerca & & \\
Asistir a las consultas usted toma & 49 & 24 \\
Tomar un taxi & 67 & 38,2 \\
Tomar una moto taxi & 46 & 22,5 \\
Tomar una buseta & 28 & 13,7 \\
Caminar & 11 & 5,4 \\
\hline Silla de ruedas & 3 & 1,5 \\
\hline Bicicleta & & \\
\hline Tiempo en asignación de citas medicas & 41 & 20,1 \\
\hline Entre 1-3 días & 50 & 24,5 \\
Entre 4-7 días & 113 & 55,4 \\
\hline Más de 7 días & & \\
\hline
\end{tabular}

Fuente: Resultados del estudio

Asociación estadística entre los factores estudiados y la utilización de servicios de salud preventivos.

- Factores asociados a la asistencia a consulta médica en el último año entre los participantes.

En los hombres con discapacidad motora que tienen pareja, la probabilidad de asistir a una consulta médica en el último año aumenta hasta 1,9 veces más que en quienes no tienen pareja $(\mathrm{OR}=1,870$; I.C. $95 \%=$ 1,020-3,428); así mismo, tener estudios superiores a la secundaria aumenta esta probabilidad $(\mathrm{OR}=2,464$; I.C. $95 \%=1,071-5,665$ y OR=3,287).

Aspectos como estar satisfecho con la comodidad y la amplitud de la sala de espera (OR=2,806; I.C. $95 \%=$
$1,375-5,727)$ y las instalaciones y equipamiento del centro médico $(\mathrm{OR}=2,956$; I.C. $95 \%=1,476-5,919)$ aumentan la probabilidad de asistir al médico en el último año; así como tener cerca el centro de salud $(\mathrm{OR}=2,186$, I.C. $95 \%=1,119-4,269) .($ Ver Tabla 4)

\section{- Factores asociados a la inscripción a programas de promoción y prevención entre los partici- pantes.}

En los participantes tener pareja (OR=3,210; I.C. $95 \%$ $1,087-9,478)$ y estar satisfecho con las instalaciones y equipamiento del centro de salud $(\mathrm{OR}=5,207$; I.C. $95 \% 1,754-15,454)$ aumenta la probabilidad de estar inscritos a un programa de promoción y prevención (Ver Tabla 4). 
ISSN-PRINT

1794-9831

E-ISSN 2322-7028

Vol. 16 No. 1

Ene - Abr 2019

Cúcuta, Colombia
Tabla 4. Factores asociados a la asistencia a consulta médica en el último año e inscripción a programas de promoción y prevención entre los participantes.

\begin{tabular}{|c|c|c|c|}
\hline \multirow{2}{*}{ Asistencia a consulta médica en el último año } & \multirow{2}{*}{ OR } & \multicolumn{2}{|c|}{ I.C. $95 \%$} \\
\hline & & Lìmite Inferior & Lìmite Superior \\
\hline Vivir en el área urbana & 0,987 & 0,511 & 1,905 \\
\hline Tener pareja & 1,870 & 1,020 & 3,428 \\
\hline Estudios superiores a la secundaria & 2,464 & 1,071 & 5,665 \\
\hline Ser de estrato 2 en adelante & 1,957 & 0,802 & 4,775 \\
\hline Tener trabajo & 1,054 & 0,586 & 1,899 \\
\hline Tener ingreso personal & 1,339 & 0,743 & 2,412 \\
\hline Ser del régimen contributivo & 0,627 & 0,136 & 2,885 \\
\hline Convivir con 4 o más personas & 0,234 & 0,021 & 2,623 \\
\hline Familia normofuncional & 0,417 & 0,229 & 0,759 \\
\hline Tener apoyo social normal & 3,287 & 1,786 & 6,048 \\
\hline Estar satisfecho con el tiempo que le dedica su médico & 0,807 & 0,438 & 1,486 \\
\hline Estar satisfecho con la solución que el médico/enfermera le da & 0,820 & 0,452 & 1,491 \\
\hline Estar satisfecho con el interés del médico en explicarle su dolencia & 0,933 & 0,517 & 1,684 \\
\hline Estar satisfecho con la Información proporcionada sobre el tratamiento & 0,804 & 0,445 & 1,452 \\
\hline Estar satisfecho con el trato recibido por el resto de personal & 1,514 & 0,833 & 2,749 \\
\hline Estar satisfecho con la disposición del personal para ayudarle & 1,709 & 0,942 & 3,101 \\
\hline Estar satisfecho con la comodidad y amplitud de la sala de espera & 2,806 & 1,375 & 5,727 \\
\hline Estar satisfecho con las instalaciones y equipamiento del centro & 2,956 & 1,476 & 5,919 \\
\hline Estar satisfecho con la señalización interna del centro & 0,427 & 0,089 & 1,417 \\
\hline Tiempo de espera desde que llegó al servicio hasta que recibió la atención menor de 30 min & 1,738 & 0,955 & 3,161 \\
\hline Tener cerca el centro de salud & 2,186 & 1,119 & 4,269 \\
\hline Demora en la asignación de citas, mayor a 7 días & 1,052 & 0,583 & 1,897 \\
\hline \multirow{2}{*}{ Inscripción a programas de promoción y prevención } & OR & \multicolumn{2}{|c|}{ I.C. 95\% } \\
\hline & & Limite Inferior & Lìmite Superior \\
\hline Vivir en el área urbana & 1,252 & 0,390 & 4,015 \\
\hline Tener pareja & 3,210 & 1,087 & 9,478 \\
\hline Estudios superiores a la secundaria & 1,168 & 0,361 & 3,781 \\
\hline Ser de estrato 2 en adelante & 1,121 & 0,304 & 4,142 \\
\hline Tener trabajo & 0,435 & 0,155 & 1,226 \\
\hline Tener ingreso personal & 0,917 & 0,339 & 2,482 \\
\hline Ser del régimen contributivo & 0,914 & 0,875 & 0,954 \\
\hline Convivir con 4 o más personas & 5,781 & 0,497 & 67,276 \\
\hline Familia normofuncional & 0,797 & 0,283 & 2,246 \\
\hline Tener apoyo social normal & 1,000 & 0,365 & 2,742 \\
\hline Estar satisfecho con el tiempo que le dedica su medico & 3,125 & 0,868 & 11,248 \\
\hline Estar satisfecho con la solución que el médico/enfermera le da & 2,594 & 0,815 & 8,251 \\
\hline Estar satisfecho con el interés del médico en explicarle su dolencia & 2,088 & 0,708 & 6,162 \\
\hline Estar satisfecho con la Información proporcionada sobre el tratamiento & 2,180 & 0,739 & 6,431 \\
\hline Estar satisfecho con el trato recibido por el resto de personal & 1,572 & 0,532 & 4,645 \\
\hline Estar satisfecho con la disposición del personal para ayudarle & 1,643 & 0,556 & 4,855 \\
\hline Estar satisfecho con la comodidad y amplitud de la sala de espera & 2,632 & 0,966 & 7,171 \\
\hline Estar satisfecho con las instalaciones y equipamiento del centro & 5,207 & 1,754 & 15,454 \\
\hline Estar satisfecho con la señalización interna del centro & 0,163 & 0,042 & 1,823 \\
\hline Tiempo de espera desde que llegó al servicio hasta que recibió la atención menor de 30 min & 1,678 & 0,612 & 4,596 \\
\hline Tener cerca el centro de salud & 1,836 & 0,675 & 4,992 \\
\hline Demora en la asignación de citas mayor a 7 días & 0,694 & 0,257 & 1,878 \\
\hline
\end{tabular}

Fuente: Resultados del estudio 


\section{Discusión}

La discapacidad es un problema de salud pública según la Organización Mundial de la Salud (OMS), porque afecta de manera significativa la calidad de vida de las personas que viven con esta condición (3). El acceso a los servicios de salud es en la actualidad uno de los principales retos de los sistemas de salud, así como su utilización por los diferentes grupos sociales, lo que demuestra la capacidad que tienen para ofrecer los recursos necesarios y dar respuesta a las necesidades de las personas (28).

Para el acceso y la utilización de los servicios de salud las personas se enfrentan a diferentes barreras, de hecho "los estudios indican la existencia de barreras relacionadas con la accesibilidad, referidas a la población (cobertura del seguro, ingresos o educación) y a las características de los servicios (accesibilidad geográfica, asuntos administrativos o calidad)" (28).

De igual forma, la accesibilidad y la utilización de los diferentes servicios, en especial los sanitarios, es limitada para la población con discapacidad motora, más cuando se debe tener en cuenta que estas personas son las que tienen mayor riesgo de afecciones secundarias y más comportamientos que ponen en riesgo su salud. Además, son quienes menos utilizan los servicios de salud preventivos, debido a que desconocen la existencia de programas de promoción y prevención, que son gratuitos y están disponibles para toda la población en general. Así mismo, esta población percibe y se enfrenta a barreras u obstáculos que les restringen la oportunidad de disfrutar plenamente de sus derechos en salud $(12,29)$.

Es importante subrayar, que en el presente estudio se realizó una caracterización con diversas variables. En cuanto a los factores sociodemográficos se encontró que la edad promedio de los participantes fue de 45 años, llama la atención que se hallan aún en edad productiva. Durante la edad adulta las personas toman la decisión de permanecer solteras o tener pareja, en el presente estudio se encontró que el 45\% (90) de los participantes tenían pareja, esto difiere de lo encontrado por Cardona y colaboradores (30) quienes indican que más del $50 \%$ de la población con algún tipo de limitación se encontraba soltera. El ser humano atraviesa por numerosos cambios personales, familiares y laborales, es por eso que durante la etapa adulta se generan una serie de acontecimientos que marcan el paso de la adolescencia a esta nueva etapa, como el establecimiento de relaciones afectivas serias, estables y formales, que conducen a tomar la decisión de permanecer soltero o casarse. Es decir, que se erige una organización familiar, que supone para el individuo un apoyo frente a cualquier situación que se presente en su vida (31).

Por otra parte, un bajo nivel educativo se asocia directamente con la posibilidad de conseguir trabajo y se refleja en los ingresos de la persona con vinculación laboral, ya sea por estar empleada o por hacerlo de manera independiente. Desde esta perspectiva, se encontró que solo el $27 \%$ de los participantes cursaron el bachillerato completo y menos del $5 \%$ tuvo un preparación tecnológica y universitaria; más del $50 \%$ trabajaba, pero menos de este porcentaje recibía un salario mínimo legal vigente SMLV, ubicándose en el estrato socioeconómico 1; estos resultados son similares a los encontrados en la política pública de discapacidad del distrito de Cartagena: "Claves para la participación con inclusión social 20102015 " (9), donde el 45,87\% de personas identificadas con discapacidad carece de nivel de escolaridad, el ingreso promedio mensual alcanza la suma de $\$ 178.183$ $\mathrm{m} / \mathrm{c}$, y el 96,6\% de la población con discapacidad de la base de datos del SISBEN se encuentra en los niveles 1 y 2. Por tanto, se evidencia que las personas con discapacidad no tienen educación superior o secundaria y un mayor índice de analfabetismo, esto se relaciona directamente, entre otros factores con el aspecto físico, el cual se asocia a un índice de inactividad más alto convirtiéndose en una condición que predispone a la exclusión de esta población en el mercado laboral y en la sociedad en general (30). Todo lo anterior se traduce en el círculo de pobreza y vulnerabilidad al cual se encuentra expuesta esta población, que en su gran mayoría recibe menos de un SMLV y que no satisface sus necesidades básicas (32).

Diferentes autores identifican el factor económico como una barrera no sólo para la inclusión social de las personas con discapacidad sino también para el acceso de éstas a los servicios de salud. Así se refleja en el municipio de Liborina-Antioquia donde el $80 \%$ de la población con discapacidad no cuenta con los recursos económicos para acceder a servicios de atención y rehabilitación en salud (33).

Con relación a la afiliación al sistema de seguridad social y la frecuencia de utilización de los servicios de salud, el 77,4\% (158) se encontraban afiliados al régimen subsidiado y el $69 \%$ (141) ha asistido a una consulta 
ISSN-PRINT

1794-9831

E-ISSN 2322-7028

Vol. 16 No. 1

Ene - Abr 2019

Cúcuta, Colombia para el cuidado de su salud en los últimos 12 meses, situación muy parecida a la encontrada en el estudio de Gómez y González (32), donde el 72,5\% son personas afiliadas al régimen subsidiado y el $72,6 \%$ ha asistido a los servicios de atención general en salud en el último año. De igual forma, el régimen subsidiado en salud constituye el mecanismo a través del cual la población de escasos recursos accede a los servicios de salud; teniendo en cuenta que el nivel socioeconómico se relaciona de manera directa con el régimen en salud de las personas, por tanto este grupo poblacional pertenecerá al régimen subsidiado de salud $(34,35)$.

En cuanto al tipo de familia, el 29,4\% (60) pertenece a una familia nuclear, esto coincide con lo encontrado por Gómez y González (32), donde el 38,9\% son personas que pertenecen a familias nucleares y además son jefe de hogar. En lo que se refiere a la funcionalidad familiar, el 34\% (69) considera tener algún grado de disfuncionalidad en su familia, resultados similares a los encontrados en el estudio de Carrasquilla y colaboradores (36) en donde el 37,5\% (64.414) de las personas con discapacidad perciben actitudes negativas de sus familias. La familia es la unidad básica de la sociedad, juega unos papeles importantes en el proceso de afrontamiento de situaciones de salud difíciles, en este caso se convierte en el principal apoyo para una persona con discapacidad, permitiéndole a esta y a todo el núcleo familiar crecer con autonomía, autoestima y seguridad (37). Sin embargo todas las familias experimentan cambios que generan eventos estresantes, lo cual no es una excepción para las familias de las personas con discapacidad, quienes se enfrentan a diversas situaciones que se reflejan en las relaciones intrafamiliares llevando incluso a la disfunción familiar y/o disgregación de la familia (8).

También, se ha encontrado evidencia sobre la relación entre la satisfacción, la utilización y el acceso a los servicios de salud, la conducta del paciente y la continuidad del uso de estos servicios. En este estudio se encontró que las personas con discapacidad motora estaban insatisfechas con algunas características del servicio, como infraestructura, señalización, comodidad y acciones realizadas por el personal de salud. Estos resultados coinciden con los encontrados por Pereira, quien indicó que las personas con discapacidad física se enfrentan a diferentes barreras para acceder a los servicios de salud tales como: rampas, pasamanos, escalones, pisos y puertas e incluso equipo médico, que no cumplen con las normas adecua- das para la libre movilización, así como la falta de una buena señalización visual para encontrar estos servicios (38).

Así mismo, Floyd señaló que en promedio el $82 \%$ de las personas les gustaría mejorar las instituciones de salud, parques, avenidas, carreteras y otros entornos públicos y privados, ya que esto facilitaría una mejor movilidad, lo cual permitiría participar en más actividades, así como también aumentar la búsqueda de servicios, especialmente aquellos relacionados con la salud (5). Las barreras arquitectónicas presentes en los servicios de atención primaria en salud determinan la forma en que las personas con discapacidad física perciben la calidad de los servicios que reciben y la satisfacción con los mismos (39).

Las dificultades y limitaciones que enfrentan las PcD motora aumentan en situaciones en las que la accesibilidad no se garantiza, lo que afecta no solo el uso de los servicios de salud sino también la autoestima, desarrollo y participación social del individuo. Se encontró que más de la mitad de los participantes tenía ubicado el centro de salud lejos, esto implicaba utilizar un medio de transporte aunado a las dificultades propias de su limitación física. Al llegar al centro de salud tenían que esperar un tiempo superior a 30 minutos y el tiempo de asignación para una nueva cita era superior a 7 días, condiciones que dificultan el acceso a estos servicios. Los datos anteriores coinciden con los resultados encontrados por Vargas, quien señala que la accesibilidad geográfica y el tiempo de espera son barreras que afectan la prestación de servicios (40).

Conviene destacar, que las grandes distancias y desplazamiento que deben recorrer las personas para acceder a los servicios de salud se convierte en una barrera de acceso teniendo en cuenta la carga económica que esto demanda (41). Para las personas con discapacidad motora esta situación no es ajena, teniendo en cuenta sus características sociodemográficas y que el factor económico ha sido identificado como una barrera de acceso y uso de los servicios de salud. Desde esta perspectiva , Fontova ha identificado cómo el tiempo de espera es un factor importante para las personas, ya que estas esperan que se les preste tratamiento rápido y piensan que habrá un médico esperándolos en el momento que entren al servicio (42). Con base a esto el tiempo de espera se convierte en un factor determinante para que el individuo esté satisfecho con los servicios a los cuales está accediendo. 
Se debe especificar que los factores asociados a la utilización de los servicios de salud preventivos en adultos con discapacidad motora son: tener pareja, estudios superiores a la secundaria, tener un apoyo social normal, tener cerca el centro de salud y estar satisfecho con las instalaciones, equipos del centro, comodidad y amplitud de la sala de espera. Además el tener una pareja, un nivel de educación superior a la secundaria y apoyo social normal, conlleva a tener conductas positivas con respecto a la salud, lo que se traduce en la utilización de los servicios de salud preventivos. Al referirnos a la satisfacción de las personas con los servicios de salud, se habla de la calidad del servicio ofrecido y del deber de subsanar las necesidades de salud del usuario; por tanto, las personas que se sientan satisfechas con la prestación de servicios de salud, continuarán haciendo uso de estos, mantendrán el vínculo con los entidades de salud, cumplirán con las consultas asignadas, estarán motivadas y sentirán el deseo de participar de manera efectiva y con compromiso en su propio cuidado, así como también aportarán información valiosa relacionada con la prestación de los servicios $(13,43)$.

A pesar de que los participantes del estudio manifestaron asistir a consultas para el cuidado de su salud, indicaban como principales razones para no utilizarlos el presentar un caso leve y que el servicio quedaba lejos. Todo esto permite concluir que aún existiendo servicios que apuntan a prevenir la aparición de enfermedades no derivadas de la discapacidad motora, y promover acciones que aumenten un mejor estado de salud, la utilización de servicios de salud preventivos por esta población es muy bajo.

La prestación de servicios de salud a las personas con discapacidad, además de ser un servicio social, es un derecho humano fundamental. Es importante tener en cuenta que para garantizar el acceso y utilización de estos se requiere que los bienes, servicios y oportunidades de salud sean adecuados para toda la población sin importar sus condiciones físicas, y para esto es necesario conocer los factores que hacen que estas personas no utilicen los servicios de salud disponibles (7).

La principal fortaleza de esta investigación fue abarcar una población representativa de hombres con discapacidad motora de la localidad 2 de Cartagena, lo que permitió identificar la poca utilización de los servicios de salud preventivos por parte de este grupo poblacional.
Entre las limitaciones del estudio se encuentran que los adultos con discapacidad es una población difícil de intervenir; en repetidas ocasiones no desearon participar, alegando sentirse utilizados como objetos de estudio, más cuando los resultados obtenidos no fueron socializados con ellos, ni percibieron que les ayudaran a mejorar sus condiciones.

\section{Conclusiones}

El grupo de estudio estuvo conformado por hombres en edad productiva, casados o en convivencia con una pareja, con bajo nivel académico, vinculados a una actividad laboral de manera independiente, un alto porcentaje pertenece al estrato socioeconómico 1 , están afiliados al régimen subsidiado en salud y más del $50 \%$ no están inscritos a programas de promoción de la salud y prevención de la enfermedad. Se percibe poca frecuencia de asistencia a la realización de exámenes de salud preventivos, examen de agudeza visual y consulta odontológica en los últimos 6 meses. Igualmente, se observa una alta insatisfacción en aspectos relacionados con la prestación de los servicios de salud como: infraestructura, señalización, comodidad y acciones realizadas por el personal de salud. Estos aspectos impactan de forma negativa en la accesibilidad y uso continuo de estos servicios.

Factores como la ubicación del centro de salud, accesibilidad geográfica, los tiempos para recibir la atención médica y para la asignación de una nueva cita médica son determinantes para que el individuo acceda y esté satisfecho con los servicios de salud.

Por otra parte, el entorno familiar y el soporte que se deriva de tener pareja, para las personas con discapacidad, se convierte en uno de los principales determinantes a la hora de buscar atención médica para el cuidado y la conservación de la salud. El efecto que ejerce la pareja y la familia en los hombres con discapacidad motora, permite que participen de los programas preventivos que se ofrecen desde las instituciones sanitarias, factor que fue evidente en este estudio y que se configura como uno de los aspectos más importantes para el cumplimiento de las citas.-

Los resultados encontrados en este estudio evidencian la necesidad de que las instituciones prestadoras de servicios de salud fortalezcan, organicen y ofrezcan programas de promoción y prevención que permitan satisfacer las necesidades de este grupo pobla- 
ISSN-PRINT

1794-9831

E-ISSN 2322-7028

Vol. 16 No. 1

Ene - Abr 2019

Cúcuta, Colombia cional, así como también mejorar las condiciones que garanticen la disponibilidad y acceso de acuerdo a la normatividad actual vigente.

Finalmente, es importante tener en cuenta que, a pesar de los riesgos no derivados de la discapacidad motora, la utilización de los servicios de salud preventivos por la población estudiada es muy baja. En general, el empleo de los servicios de salud se ve influenciada por el apoyo de la familia, el tener pareja y el estar o no satisfecho.

\section{Conflicto de intereses}

Las autoras declaran no tener ningún conflicto de interés.

\section{Referencias Bibliográficas}

1. Organización Mundial de la Salud (OMS), Banco Mundial. Resumen Informe Mundial Sobre la Discapacidad. [Internet] Ginebra 2011[consultado 21 de Diciembre de 2017]; Disponible en: http://www. who.int/disabilities/world_report/2011/summary_es.pdf

2. Serrano C, Ramírez C, Miranda A, Patricia J, Camargo R, Vivian L, et al. Barreras contextuales para la participación de las personas con discapacidad física: Discapacidad y barreras contextuales. Revista de la Universidad Industrial de Santander Salud. 2013; 45(1):41-51.

3. Gómez Aristizábal LY, Avella Tolosa A, Morales LA. Observatorio de Discapacidad de Colombia. Rev. Fac. Nac. Salud Pública. 2015; 33(2): 277-285.

4. Secretaria de educación del estado de Veracruz. Discapacidad Motriz. Dirección general de educación especial. 2008.

5. Floyd M, Zambrano J, Antó A, Jiménez C, Solórzano C, Díaz A. Identificación de las barreras del entorno que afectan la inclusión social de las personas con discapacidad motriz de miembros inferiores. Salud Uninorte. 2012; 28(2): 227-237.

6. República de Colombia. Ministerio De Salud y Protección Social. Plan decenal de salud pública. 2012-2021: En Colombia la salud la construyes tú [Internet] 2012. [consultado 21 de Diciembre de 2017]. Disponible en: https://www.minsalud.gov.co/Documentos\%20y\%20Publicaciones/Plan\%20 Decenal\%20-\%20Documento\%20en\%20consulta\%20para\%20aprobaci\%C3\%B3n.pdf

7. Osorio Vega JL. Reportes del Registro de Localización y Caracterización de la Población con Discapacidad del Distrito de Cartagena 2015. Departamento Administrativo Distrital de Salud- DADIS. [Internet]. Cartagena, Colombia [consultado 21 de diciembre de 2017]. Disponible en: http://www. dadiscartagena.gov.co/images/docs/pau/reporte_rlcpd_12_2015.pdf

8. Gómez J, González C. Discapacidad en Colombia: reto para la inclusión en capital humano. Bogotá: Fundación Saldarriaga Concha. 2010:1-6.

9. Consejo Distrital de Política Social de Cartagena de Indias. Política Pública De Discapacidad En El Distrito De Cartagena "Claves Para La Participación Con Inclusión Social” 2010-2015. [Consultado 21 de diciembre de 2017]. Disponible en: http://enmodoin.com/wp-content/uploads/2015/11/ppddcartagena.pdf

10. Organización Mundial de la Salud (OMS). Discapacidad y Salud. [Internet] Washington: OMS; 2014. [Consultado 20 de marzo de 2014]. Disponible en: http://www.who.int/mediacentre/factsheets/fs352/ es/

11. República de Colombia. Ministerio de salud y protección social. Análisis de Situación de Salud de Poblaciones Diferenciales Relevantes. [Internet] Ministerio de Salud y Protección Social Dirección de Epidemiología y Demografía Grupo ASIS. [Consultado 22 de marzo de 2014]. Disponible en: https:// www.minsalud.gov.co/Documentos $\% 20 \mathrm{y} \% 20$ Publicaciones/An $\% \mathrm{C} 3 \% \mathrm{~A} 1 \mathrm{lisis} \% 20 \mathrm{de} \% 20$ poblaciones\%20diferenciales.pdf

12. Martínez Rozo AM, Uribe Rodríguez AF, Velázquez González HJ. La discapacidad y su estado ac- 
tual en la legislación colombiana. Revista Duazary. [Internet]. 2015 [Consultado 11 de octubre de 2017]; 12(1):49-58. Disponible en: http://revistas.unimagdalena.edu.co/index.php/duazary/article/ view/1398/799

13. Organización de las Naciones Unidas (ONU). Convención sobre los derechos de las personas con discapacidad [Internet]. [Consultado 11 de octubre de 2017]. Disponible en: https://www.un.org/spanish/ disabilities/default.asp?id $=497$

14. Vargas J, Molina G. Acceso a los servicios de salud en 6 ciudades de Colombia: limitaciones y consecuencias. Rev Fac Nac Salud Pública. 2009; 27(2):121-130

15. República de Colombia. Resolución 3165 de 1996. Adopta lineamientos de atención en salud para las personas con deficiencias, discapacidades y minusvalías. Bogotá: ministerio de salud y protección social. 1996

16. República de Colombia. El Presidente de la República de Colombia. Decreto 1538 de 2005. Por el cual se reglamenta parcialmente la Ley 361 de 1997. [Internet]. Bogotá: 2005 [Consultado 11 de octubre de 2017]. Disponible en: http://www.alcaldiabogota.gov.co/sisjur/normas/Norma1.jsp?i=16540

17. Organización Internacional de Trabajo, Organización Mundial de la Salud, Organización de las Naciones Unidas para la Educación, la Ciencia y la Cultura. (2004). Estrategia para la rehabilitación, la igualdad de oportunidades, la reducción de la pobreza y la integración social de las personas con discapacidad. Documento de posición conjunta, Suiza: OIT, OMS, Unesco, p. 3

18. Correa ML. Panorama de la protección jurisprudencial a los derechos humanos de las personas con discapacidad en Colombia. Universitas. 2009; 58(118): 115-139.

19. Hernández JJ, Hernández UI. Una aproximación a los costos indirectos de la discapacidad en Colombia. Revista de Salud Pública .2009; 7(2): 130-144.

20. Gudlavalleti M, Neena J, Allagh K, Sagar J, Kamalakannan S, Ramachandra S. Access to health care and employment status of people with disabilities in South India, the SIDE (South India Disability Evidence) study. BMC public health. 2014; 14(1):1.

21. Hernández-Quevedo C, Jiménez-Rubio D. Inequidad en la utilización de servicios sociosanitarios en España para las personas discapacitadas. Gaceta Sanitaria. 2011; 25:85-92.

22. Bellón JA, Delgado A, Luna JD, Lardelli P. Validez y fiabilidad del cuestionario de función familiar Apgar-familiar. Aten Primaria 1996; 18 (6): 289-295.

23. Bellón JA, Delgado A, Luna J, Lardelli P. Validez y fiabilidad del cuestionario de apoyo social funcional Duke-UNC-11. Aten Primaria, 1996; 18: 153-63.

24. Cuellar I. Dresch V. Validación del cuestionario de Apoyo Social Funcional Duke-UNK-11 en personas cuidadoras. Revista Iberoamericana de Diagnóstico y Evaluación - e Avaliação Psicológica. 2012; 2(34): 89-101.

25. Varela J, Rial A, Garcia E. Presentación de una escala de satisfacción con los servicios sanitarios de atención primaria. Psicothema.2003; 15:656-661

26. República de Colombia. Ministerio de Salud. Resolución No 008430 de 1993, octubre 4, por la cual se establecen las normas científicas, técnicas y administrativas para la investigación en salud. [Internet]. Santa Fe de Bogotá: El Ministerio; 1993 [consultado 29 de enero de 2014]. Disponible en: https://www. minsalud.gov.co/sites/rid/Lists/BibliotecaDigital/RIDE/DE/DIJ/RESOLUCION-8430-DE-1993.PDF

27. Serrano D, Linares A. Principios éticos de la investigación biomédica en seres humanos: aplicación y limitaciones en américa latina y el caribe. Bol of Sanit Panam [Internet] 1990. [Consultado 10 de agosto de 2014]; 108(5-6):489-499. Disponible en: http://iris.paho.org/xmlui/bitstream/handle/123456789/16826/v108n\%285-6\%29p489.pdf?sequence $=1 \&$ isAllowed $=\mathrm{y}$

28. Restrepo-Zea JH, Silva-Maya C, Andrade-Rivas F, VH-Dover, R. Acceso a servicios de salud: análisis de barreras y estrategias en el caso de Medellín, Colombia. Rev. Gerenc. Polít. Salud. 2014; 13(27): 
ISSN-PRINT

1794-9831

E-ISSN 2322-7028

Vol. 16 No. 1

Ene - Abr 2019

Cúcuta, Colombia
242-265.

29. Kroll T. Jones G. Kehn M. Neri M. Barriers and strategies affecting the utilisation of primary preventive services for people with physical disabilities: a qualitative inquiry. Health and Social Care in the Community. 2006; 14(4): 284-293.

30. Cardona Arango D, Agudelo Martínez A, Restrepo Molina L, Segura Cardona ÁM. Educación, salud y empleo de la población con algún tipo de limitación. Medellín, 2008. Revista Hacia la Promoción de la Salud. 2012; 17(1):77-91.

31. Soto R. Factores que intervienen en la elección de pareja de jóvenes mexicanos. Revista REDES. 2015(32).

32. Gómez J, González C. Discapacidad en Colombia: reto para la inclusión en capital humano. Bogotá: Fundación Saldarriaga Concha. 2010:1- 6.

33. Consejo municipal de Liborina. Proyecto de política pública de discapacidad. Liborina. 2008.

34. Ministerio de salud y protección social. Régimen subsidiado.

35. Díaz C, Zapata Y, Aristizábal J. Acceso A los servicios preventivos en los regímenes contributivo y subsidiado de salud en un barrio estrato dos de la ciudad de cali. Rev. Gerenc. Polit. Salud, Bogotá (Colombia). 2011; 10 (21): 153-175.

36. Carrasquilla G, Martínez S, Latorre M, Garcia S, Rincon C, Olaya C, Castro R, et al. La discapacidad en el contexto del Sistema General de Seguridad Social en Salud en Colombia: Lineamientos, epidemiología e impacto económico. Fundación Saldarriaga Concha y Fundación Santa Fe de Bogotá. 2009 [Internet]. 2008 [Consultado 06 de agosto de 2014] Disponible en: https://mesadesplazamientoydiscapacidad.files.wordpress.com/2010/05/doc-fundac-2009.pdf

37. Cid M, Montes R, Hernandez O. La familia en el cuidado de la salud. Revista Médica Electrónica. 2014; 36(4):462-72.

38. Pereira E, Salvador M, Harter R. Barriers related to screening examinations for prostate cancer. Revista Latino-Americana de Enfermagem. 2011; 19:73-80.

39. Ekwall A, Gerdtz M, Manias E. The influence of patient acuity on satisfaction with emergency care: perspectives of family, friends and carers. Journal of Clinical Nursing. 2008; 17(6):800-9.

40. Vargas I, Vázquez M, Mogollón A. Acceso a la atención en salud en Colombia. Rev. Salud pública. 2010; 12 (5): 701-712.

41. Vargas J, Molina G. Acceso a los servicios de salud en seis ciudades de Colombia: limitaciones y consecuencias. Facultad Nacional de Salud Pública: El escenario para la salud pública desde la ciencia. 2009; 27(2):121-30

42. Fontova A, Juvinyá D, Suñer R. Influencia del tiempo de espera en la satisfacción de pacientes y acompañantes. Revista de Calidad Asistencial. 2015; 30 (1):10-6.

43. Pérez CM, Ortiz Reyes RM, Llantá Abreu M del C, Peña Fortes M, Infante Ochoa I. La evaluación de la satisfacción en salud: un reto a la calidad. Rev Cubana Salud Pública [Internet]. 2008 [Consultado 11 de octubre de 2017]; 34 (4). Disponible en: http://scielo.sld.cu/scielo.php?script=sci_arttext\&pid=S0864$34662008000400013 \& \operatorname{lng}=\mathrm{es}$ 